\title{
Research of the conductivity of organic and inorganic media in multi-angle multi-frequency electrical impedance tomography
}

\author{
Grayr Aleksanyan ${ }^{1 *}$, Ivan Shcherbakov ${ }^{1}$, Artem Kucher $^{1}$, and Maria Priyma ${ }^{1}$ \\ ${ }^{1}$ SRSPU (NPI), Department of Information and Measuring Systems and Technologies, 346428 Novocherkassk, Russia
}

\begin{abstract}
The work is devoted to the study of the impedance of organic and inorganic media containing inhomogeneities at different frequencies of the injecting current in order to determine the conditions for using the device for multi-angle multi-frequency electrical impedance tomography. The paper provides a method of obtaining data and analysis of the obtained measurement results. Based on the analysis, the dependences of the integral parameter of the change in the potential difference correlating with the total impedance on the frequency of the injected current are determined. To estimate the repeatability of the obtained measurement results, an analysis of the measurement data array was carried out by calculating the standard (root-mean-square) deviation for each obtained in measurement value. Based on the analysis of deviations, the dependences of the standard deviation of the obtained integral parameters on the frequency of the injected current are also determined.
\end{abstract}

\section{Introduction}

Electrical impedance tomography (EIT) is a noninvasive method for visualizing and evaluating the conduction field of internal structures of objects (both technical and biological) $[1,2]$. One of the new directions in the development of EIT is multi-angle multifrequency electric impedance tomography (MMEIT). In the course of the MMEIT, the frequency of the injected current applied to the biological object (BO) plays an important role. Due to the fact that the electrical properties of $\mathrm{BO}$ tissues depend on the frequency of the injecting current, the use of MMEIT in clinical practice is an important tool for obtaining diagnostic information. [3-17]

The aim of this work is to study the influence of the frequency of the injected current on the result of measurements, that is, on the impedance. Since devices for carrying out MMEIT operate with a limited range of load resistance, which in this case is the impedance of the $\mathrm{BO}$, the results of this work can be applied to determine the conditions for using this type of device.

\section{Materials and Methods}

\subsection{Experimental design}

To estimate the impedance, around the perimeter of the container with polyethylene non-conductive walls containing the media under study (phantom [18]), 16 electrodes from the copper-nickel alloy were placed [18], current injection was performed clockwise. To model the inhomogeneity, cylinders of $18 \mathrm{~mm}$ in diameter from non-conductive (polyethylene polymer) and conductive (stainless steel) material were placed in the media under investigation. For correct positioning of inhomoheneity samples, constant relative to electrodes, printed target mounted in a bottom of a phantom.

During the measurements a model of the hardwaresoftware complex of electrical impedance tomography [19] was used, in which the possibility of connecting a current source to any of the 16 electrodes and measuring the potential relative to a common point on any of the 16 electrodes is realized. In present study, the potential difference $\Delta \varphi$ was measured between all neighboring electrodes in pairs at frequencies $f_{I}=50 \ldots 250 \mathrm{kHz}$ in 50 $\mathrm{kHz}$ increments at the constant amplitude of the injected current $\mathrm{I}_{\mathrm{M}}=5 \mathrm{~mA}$. Since the device uses the current source developed in [20], and the impedance $\mathrm{Z}$ is calculated by the formula [4]:

$$
Z=\frac{\Delta \varphi}{I_{M}},
$$

where $\mathrm{Z}$ is the impedance value; $\Delta \varphi$ is the measured potential difference; $I_{M}$ is the amplitude of the injected current, the potential difference in a predetermined frequency range correlates with the impedance of the sample under investigation.

Potential differences $\Delta \varphi$ for all selected potentialmeasuring points is summed and normalized to obtain the integral parameter $\Theta$, which also correlates with the global impedance $\mathrm{Z}$ of the investigated object.

The measurements were carried out with two types of 'media: inorganic (tap water, total salt content of 452 $\mathrm{mg} / \mathrm{l}$, temperature of $16.8^{\circ} \mathrm{C}$ ) and organic (chicken leg quarter minced in industrial grinder to avoid metal inclusions that may affect on resulting impedance, with

\footnotetext{
* Corresponding author: graer@yandex.ru
} 
temperature of $4.2^{\circ} \mathrm{C}$ ), at an ambient temperature of $24.8^{\circ} \mathrm{C}$, atmospheric pressure of $754 \mathrm{~mm} \mathrm{Hg}$; and air humidity of $43 \%$. Photos of phantom with the placed inhomoheneity samples and electrodes connected to the measuring device are shown in Fig. 1 (a is an inorganic media with a conducting object, $\mathrm{b}$ is an inorganic media with a non-conducting object, $\mathrm{c}$ is an organic media with a conductive object, and $d$ is an organic media with a non-conducting object). Eleven repeated experiments were performed for each organic and inorganic media, conductive and non-conductive sample and frequency $f_{I}$ values with total count of experimental measurements of 220. As a result of the measurement, the average value of integral parameter $\Theta$ of repeated experiments was taken to measurement inaccuracy minimization.

To estimate the repeatability of the experimental results, the standard (root-mean-square) deviation $\sigma$ of $\Theta$, calculated by formula (2), is applied:

$$
\sigma=\sqrt{\frac{1}{n} \sum_{1}^{n}\left(x_{i}-\bar{x}\right)^{2}},
$$

where $n$ is the number of measurements,

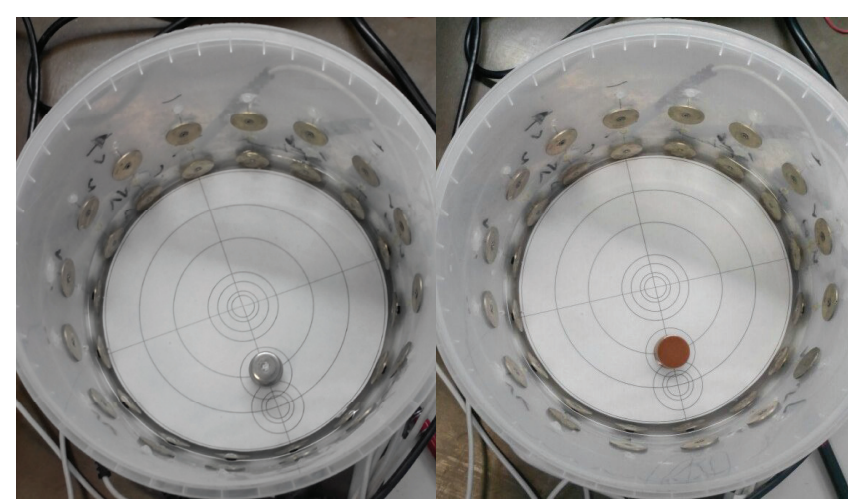

a)

b)

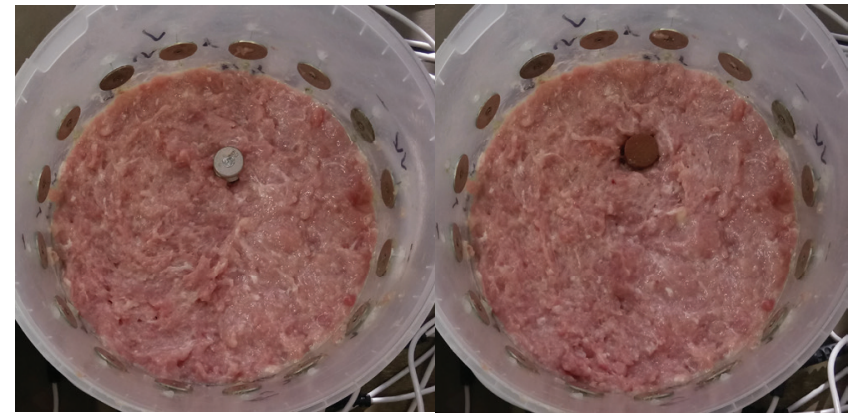

c)

d)

Fig. 1. Phantom with connected electrodes with:

a) an inorganic media with a conducting object; b) an inorganic media with a non-conducting object; c) an organic media with a conducting object; d) an organic media with a non-conducting object.

$x i$ is the value obtained in measurement,

$\bar{x}$ - the arithmetic mean, calculated by the formula:

$$
\bar{x}=\frac{1}{n} \sum_{i=1}^{n} x_{i}
$$

\section{Results and discussion}

Figure 2 shows the results of integral parameter $\Theta$, obtained from measured on electrodes potential differences, that proportional to the change in global impedance of investigated object $\mathrm{Z}$, on the frequency fi of the injected current.

Figure 2 shows the following dependencies:

- Fig. 2 (a) diagram of the dependence of $\Theta$ on fi for an inorganic media and a conductive sample;

- Fig. 2 (b) diagram of the dependence of $\Theta$ on fi for an inorganic media and a non-conducting sample;

- Fig. 2 (c) diagram of the dependence of $\Theta$ on fi for an organic media and a non-conductive sample;

- in Fig. 2 (d) diagram of the dependence of $\Theta$ on fi for an organic media and a non-conducting sample;

In order to estimate the repeatability of the research results, the results of the change in the standard (rootmean-square) deviation $\sigma$ of the integral parameter $\Theta$ from the frequency fi of the injected current $I_{M}$ are presented in Fig. 3. The following dependencies are shown in the figure:

- Fig. 3 (a) diagram of the dependence of $\sigma$ on fi for an inorganic media and a conductive sample;

- Fig. 3 (b) diagram of the dependence of $\sigma$ on fi for an inorganic media and a non-conducting sample;

- Fig. 3 (c) diagram of the dependence of $\sigma$ on fi for an organic media and a conductive sample;

- in Fig. 3 (d) a diagram of the dependence of $\sigma$ on fi for an organic media and a non-conducting sample;

Analysis of the data presented in Figures 2 shows that for the inorganic media, both with the conducting object and with the non-conducting as the inhomogeneity, with increasing fi the parameter $\Theta$ increases, which indicates an increase in global impedance $Z$.

At the same time, for an organic media, both with a conducting and non-conducting object as an inhomogeneity, the parameter $\Theta$ decreases with increasing fi, which indicates a drop in global impedance Z.

The analysis of the data obtained from the measurement of the standard deviation $\sigma$ of the obtained integral parameter $\Theta$ shown in Figures 3 indicates an increase in the deviation of measured parameters with increasing frequency in all cases both of organic and inorganic media with both conductive and nonconductive samples, which indicates an increase in the spread of the measured parameters with increasing frequency, that measurement inaccuracy may be due to an insufficiently high sampling frequency of analog to digital converter, which is part of the layout of electrical impedance tomography. In any case this effect is a subject of further studies. 


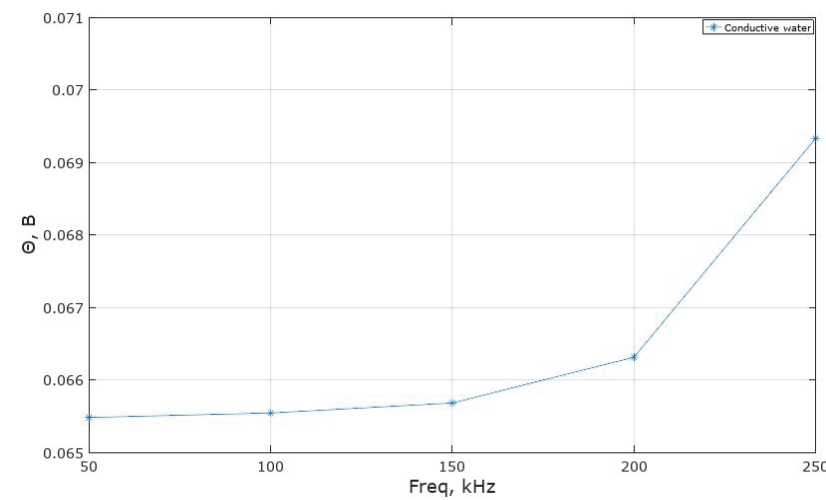

a)

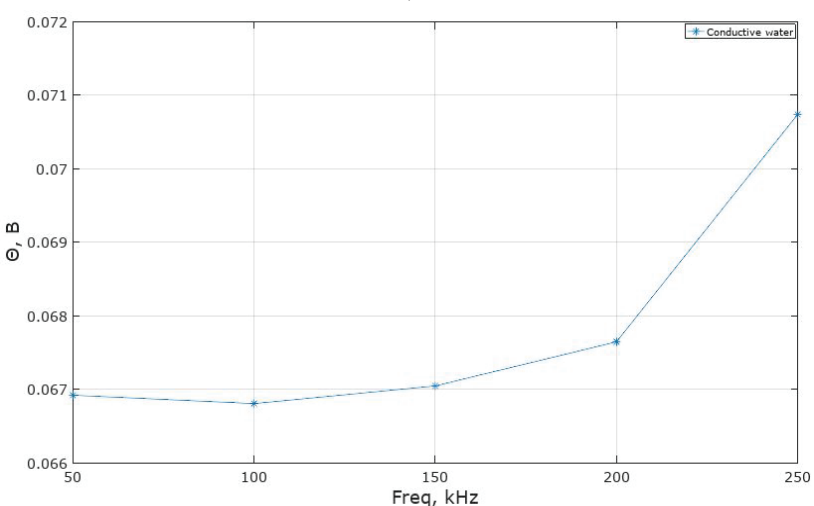

b)

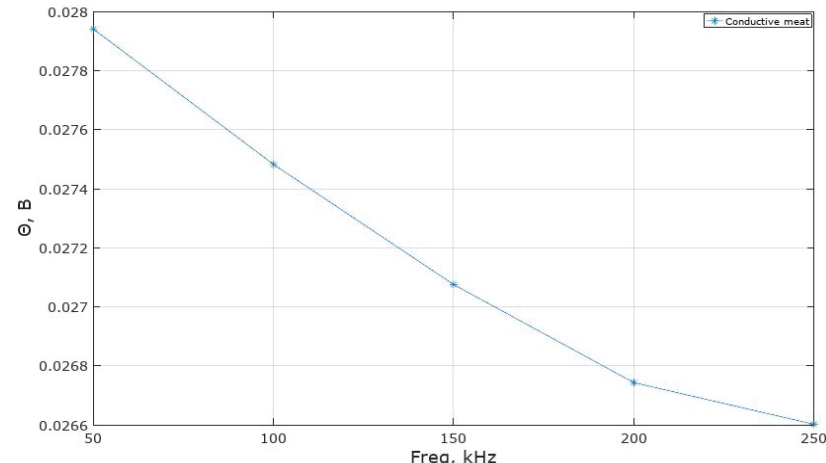

c)

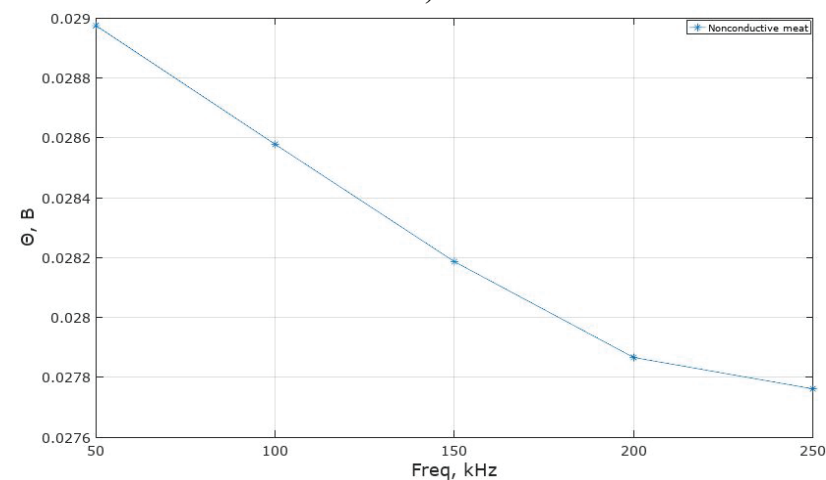

d)

Fig. 2. a) the dependence of $\Theta$ on fi for an inorganic media with a conducting object; b) the dependence of $\Theta$ on fi for an inorganic media with a non-conducting object; c) the dependence of $\Theta$ on fi for an organic media with a conducting object; d) the dependence of $\Theta$ on fi for an organic media with a non-conducting object.

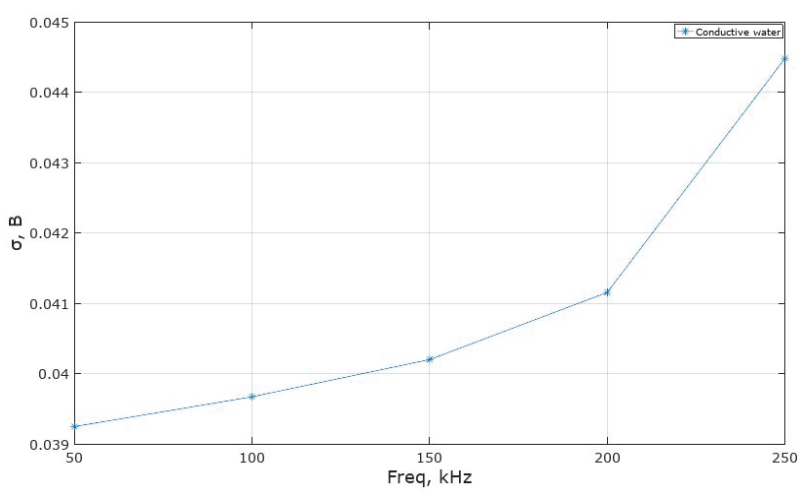

a)

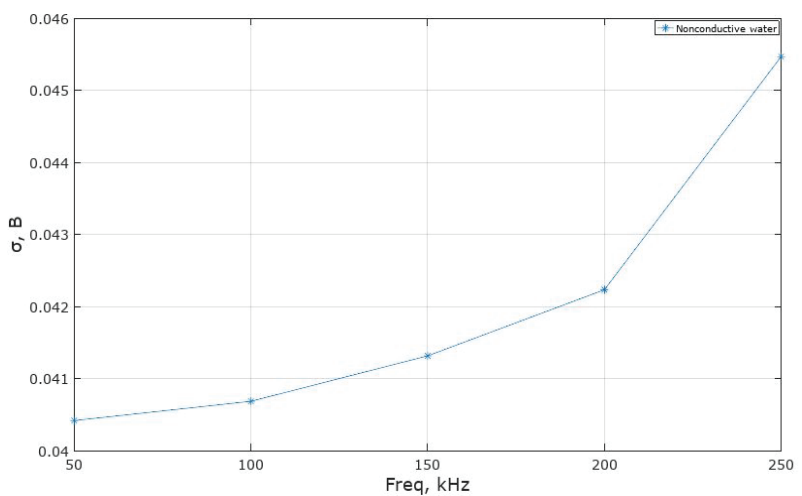

c)

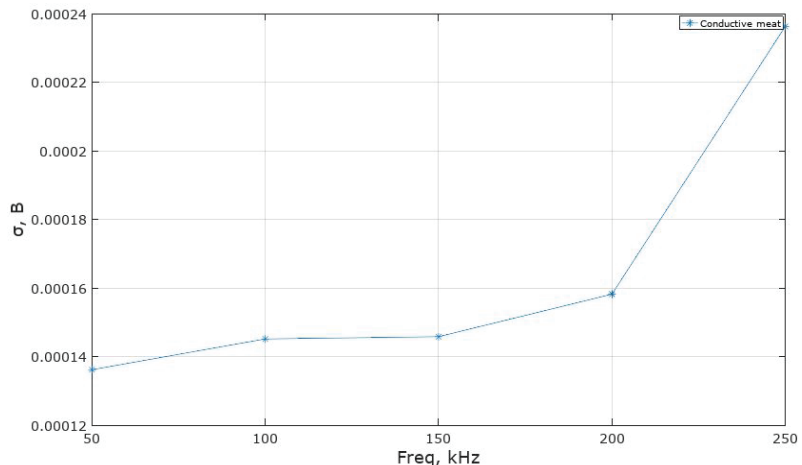

b)

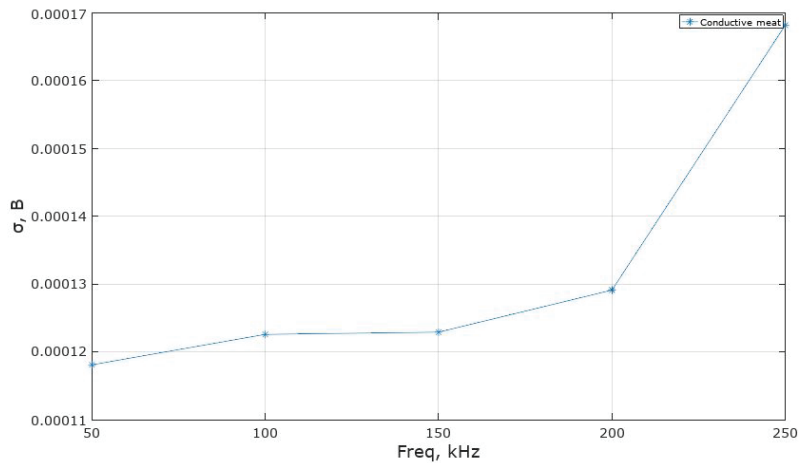

d)

Fig. 3. a) dependence of $\sigma$ on fi for an inorganic media with a conducting object; b) the dependence of $\sigma$ on fi for an inorganic media with a non-conducting object; c) the dependence of $\sigma$ on fi for an organic media with a conducting object; d) the dependence of $\sigma$ on fi for an organic media with a non-conducting object. 


\section{Conclusion}

As a result of the study, the dependences of the integral parameter $\Theta$ (that correlating with impedance Z) on frequency fi was found for variety of conductive and non-conductive samples of predetermined size and position in organic and inorganic media. Using the mock-up of the hardware-software complex of electrical impedance tomography on a phantom in the range of frequencies $\mathrm{fi}=50 \ldots 250 \mathrm{kHz}$ with a increment of 50 $\mathrm{kHz}$ and constant amplitude $\mathrm{I}_{\mathrm{M}}=5 \mathrm{~mA}$ of injected current, the values of mentioned parameter $\Theta$ were obtained.

On the basis of the measured data, the dependencies of the integral parameter $\Theta$ on the frequency fi at MMEIT are obtained.

An analysis of the dependencies obtained allows us to state that the impedance $\mathrm{Z}$ of an inorganic media with conducting and non-conducting inhomogeneities as the test sample of predetermined sizes increases slightly with increasing frequency fi of the injection current $\mathrm{I}_{\mathrm{M}}$.

At the same time, for an organic media with both conductive and non-conducting inhomogeneities as the test sample of the same sizes, the impedance $\mathrm{Z}$ decreases with increasing frequency fi of the injection current $\mathrm{I}_{\mathrm{M}}$.

The obtained dependencies show that the effectiveness of multi-angle multi-frequency electrical impedance tomography as a visualization tool for noninvasive investigation of internal structures of a biological object (which can be considered, of course, as a organic media) can be improved by varying the frequencies of the injected current.

At the same time, conducted research shows that varying the frequencies of the injected current when investigating inorganic media gives no positive effect to conductance: impedance $\mathrm{Z}$ slightly increases with increasing frequency of injection current. On other hand, such effect can give possibility to improve contrast of boundaries of reconstructed EIT-image [20] when inorganic conductive inhomoheneity is located in organic media (to clarify position and size of cavity containing liquid in a lung, or a bullet inside a body, for example).

Also conducted analysis showed a increase of standard deviation of integral parameter $\Theta$ that indicates a lower repeatability of the measurement results with increasing frequency, which is the subject of further research that will be conducted to find factors that affect this.

Work is performed within the grant of President of Russian Federation for state support of young Russian scientists MK196.2017.8 "Development of theoretical foundations and algorithms for multi-view systems are three-dimensional electrical impedance tomography for non-invasive medical imaging".

\section{References}

1. Y.S. Pecker, K.S. Brazovskii, V.Y. Usov, M.P. Plotnikov and O.S. Umansky, "Electrical impedance tomography" ("NTL" Publishing House, Tomsk, 2004)

2. D.S. Holder, Electrical Impedance Tomography. Methods, History and Applications (Institute of Physics Publishing, Bristol and Philadelphia, 2005)

3. Y. Zhou, X. Li, Measurement: J Int. Meas. Confed., 110, 27-42 (2017)

4. M.G. Crabb, Inverse Prob. in Sci. \& Eng., 25(10), 1397-1422 (2017)

5. M.G. Crabb, Inverse Prob. in Sci. \& Eng., 25(10), X (2017)

6. T.N. Tallman, J.A. Hernandez, NDT and E International, 91, 156-163 (2017)

7. G.K. Aleksanyan, N.I. Gorbatenko, V.V. Grechikhin, T.N. Phong, T.D. Lam, ARPN J of Eng. \& Appl. Sci., 11(9), 5871-5875 (2016)

8. G.K. Aleksanyan, N.I. Gorbatenko, A.I. Kucher, K.M. Shirokov, C.N. Phong, Biosciences Biotechnology Research Asia, 12, 709-718 (2015)

9. G.K. Aleksanyan, N.I. Gorbatenko, A.I. Kucher, T. N. Phong, N. M. Cuong, Int. J of Appl. Eng. Res., 10(19), 40580-40584. (2015)

10. G.K. Aleksanyan, N.I. Gorbatenko, A.D. Tarasov, Biosciences Biotechnology Research Asia, 11, 85-91 (2014).

11. G.K. Aleksanyan, A.I. Kucher, A.D. Tarasov, N.M. Cuong, C.N. Phong, Int. J of Soft Comp., 10 (6), 462-467 (2015)

12. K.S. Brazovskii, Methods and tools to evaluate the functional state of the human brain based on the electrical measurements (Dr. tehn. Sciences, Tomsk, 2015)

13. N.I. Gorbatenko, Full-scale-model testing of products made of ferromagnetic materials (Publishing House SKNTS VSH, Rostov n / D, 2001)

14. A.M. Lankin, M.V. Lankin, G. K. Aleksanyan, N.D. Narakidze, Int. J of Appl. Eng. Res., 10(3), 64896498 (2015)

15. D.V. Nikolaev, A.V. Smirnov, I.G. Bobrinskaya, S.G. Rudnev Bioimpedance analysis of the composition of the human body (Nauka, Moscow, 2009)

16. A.L. Zuev, V.Y. Mishlanov, A.I. Sudakov, N.V. Shakirov, A.V. Frolov, Russ. J of Biomechanics, 161(55), 110-120 (2012)

17. G.K. Aleksanyan, I.D. Shcherbakov, A.I. Kucher, V.V. Demyanov, J of Eng. \& Appl. Sci., 12 (8), 2129-2137 (2017)

18. G.K. Aleksanyan, N.I. Gorbatenko, A.I. Kucher, Int. J Appl. Eng. Res., 10 (19), 40580-40584 (2015)

19. G.K. Aleksanyan, A.I. Kucher, A.D. Tarasov, N.M. Cuong, C.N. Phong, Int. J Soft Comp., 10 (6), 462467 (2015)

20. G.K. Aleksanyan, I.D. Shcherbakov, A.I. Kucher, J Eng. \& Appl. Sci., 12 (3), 587-592 (2017) 